\title{
Prevalence and pattern of Iron deficiency in patient with heart failure with reduced ejection fraction
}

\author{
Aditya Mahaseth' ${ }^{1}$, Jay Narayan Shah ${ }^{2}$, Bikash Nepal' ${ }^{1}$, Biplave Karki' ${ }^{1}$, Jeet Ghimire1, Naveen \\ Kumar Pandey ${ }^{3}$, Prashant Shah ${ }^{3}$, Prahlad Karki ${ }^{4}$
}

${ }^{1}$ DM Senior Resident, Department of Cardiology, BP Koirala Institute of Health Sciences, Dharan, Nepal

${ }^{2}$ House Officer, Department of Cardiology, BP Koirala Institute of Health Sciences, Dharan, Nepal

${ }^{3}$ Associate Professor, Department of Cardiology, BP Koirala Institute of Health Sciences, Dharan, Nepal

${ }^{4}$ Professor and Head, Department of Cardiology, BP Koirala Institute of Health Sciences, Dharan, Nepal

\begin{abstract}
Background and Objectives: Iron Deficiency is the commonest nutritional deficiency worldwide, affecting more than one-third of the population, its association with Heart Failure with or without anemia is of growing interest. As iron supplementation improves prognosis in patients with Heart Failure, Iron Deficiency is an attractive therapeutic target - a hypothesis that has recently been tested in clinical studies. This study is designed to estimate the prevalence and pattern of iron deficiency (ID) in heart failure (HF) with reduced ejection fraction patients with or without anemia.
\end{abstract}

Material and methods: It was a single center hospital based cross sectional observational study. A total of 60 male and female patients with diagnosis of heart failure based on the Framingham Criteria, who gave consent for the study were included. They underwent laboratory evaluation including hemoglobin concentration, serum iron, transferrin saturation percentage, serum ferritin, total iron binding capacity. Serum ferritin $<100 \mu \mathrm{g} / \mathrm{l}$ was used to diagnose absolute ID. Functional ID was defined as a serum ferritin level of 100-300 $\mu \mathrm{g} / \mathrm{l}$ and a transferrin saturation of $<20 \%$. Anemia was defined as hemoglobin $(\mathrm{Hb})<13 \mathrm{~g} / \mathrm{dl}$ for males and $<12 \mathrm{~g} / \mathrm{dl}$ for females, based on World Health Organization definition.

Results: Using the above definitions iron deficiency was found in 28 (46.67\%) patients. 36.67\% patients had absolute iron deficiency and $10 \%$ patients had functional iron deficiency. Females had a higher non statistically significant iron deficiency than males $63.16 \%$ vs $39.02 \%$. 15 patients (48.38\%) with iron deficiency did not have anemia, and 11 (35.5\%) of those patients had absolute iron deficiency.

Conclusion: Iron deficiency is prevalent in patients with heart failure and reduced ejection fraction irrespective of anemia and hemoglobin levels. Many of those patients can have functional iron deficiency. Measurement of iron status should be a routine during workup of heart failure patients and further studies are needed to determine the prognostic value of iron status measurement and the influences of treatment of iron deficiency in heart failure patients. Many such trials are now underway.

Key words: Anemia, Heart failure, Hemoglobin, Iron Deficiency, Iron deficiency in heart failure 


\section{INTRODUCTION}

Iron Deficiency is the commonest nutritional deficiency worldwide, affecting more than one-third of the population [1]. Its association with Heart Failure with or without anemia is of growing interest [2]. Iron deficiency is an extremely common co-morbidity in patients with heart failure, affecting up to $50 \%$ of all ambulatory patients. It is associated with reduced exercise capacity and physical wellbeing and reduced quality of life [3]. Recently, iron deficiency (ID) with or without anaemia has been recognized as an emerging therapeutic target with prognostic implications [4]. Cutoff values have been identified for diagnosing iron deficiency in heart failure with reduced ejection fraction as serum ferritin $<100 \mu \mathrm{g} / \mathrm{l}$, or ferritin 100 to $300 \mu \mathrm{g} / \mathrm{l}$, with transferrin saturation of $<20 \%$ [5]. Oral iron products have been shown to have little efficacy in heart failure, where the preference is intravenous iron products. Most clinical studies have been performed using ferric carboxymaltose with good efficacy in terms of improvements in 6-min walk test distance, peak oxygen consumption, quality of life, and improvements in New York Heart Association functional class. The epidemiology, diagnosis and therapies have been extensively studied in high-income countries but are still largely unexplored in low income developing world, where infections and malnutrition are more likely to influence the situation. Current guidelines of the European Society of Cardiology for the diagnosis and treatment of HF state that all patients should be screened for iron deficiency and anaemia, a class I recommendation based on meta-analysis (level of evidence: A, because two large trials, Ferinject Assessment in Patients with Iron Deficiency and Chronic Heart Failure [FAIR$\mathrm{HF}]$ and Ferric CarboxymaltOse evaluatioN on performance in patients with IRon deficiency in combination with chronic Heart Failure [CONFIRM-HF], published positive results). Patients who remain symptomatic in New York Heart Association classes II-IV benefit from iron supplementation, preferably via the IV route[5]. Several studies with intravenous iron are ongoing; for example, the Iron or Placebo for Anaemia in Intensive Care (IRONMAN study) (NCT03037931, NCT03833336, NCT02937454, NCT03218384, NCT02642562). That study will address whether the additional use of IV iron (iron isomaltoside) on top of standard care will improve the outlook for patients with HF and iron deficiency.

Other studies are the FAIR trials. The purpose of the FAIR HF2 study is to determine whether intravenous iron supplementation (ferric carboxymaltose) reduces hospitalisation and mortality in patients with iron deficiency and HF (NCT03036462). The FAIR HFpEF study addresses whether treatment with IV iron (ferric carboxymaltose) for patients with HF with preserved ejection fraction and iron deficiency can improve exercise capacity and symptoms while being safe (NCT03074591).

Recent results of the FERRIC iron in Heart Failure (FERRIC HF II) trial showed that iron isomaltose was safe and well tolerated in patients with chronic HF and iron deficiency. They showed that iron isomaltoside was associated with faster skeletal muscle energy measured in the form of adenosine triphosphate and phosphocreatine after 2 weeks, implying better mitochondrial function. Additionally, these results showed that iron per se is an obligate component of mitochondrial enzymes that generate cellular energy in the form of adenosine triphosphate and phosphocreatine. Augmented skeletal muscle energetics might be an important 
mechanism by which iron repletion confers benefits in chronic HF. The exact mechanisms by which chronic heart failure patients develop iron deficiency are still not completely understood.[6]

Moliner et al. recently showed an interplay between raised sympathetic nervous system activity and systemic iron deficiency in patients with chronic HF and, particularly, with those biomarkers that suggest impaired iron transport (transferrin saturation <20\%) and increased iron demand (raised soluble transferrin receptor levels).[7] This impressively supports the hypothesis that iron deficiency might not just be a comorbidity, but may also be a key element in the pathophysiological sequence leading to, and promoting the progression of, chronic HF. However, many questions remain and require further research.

\section{MATERIAL AND METHODS}

This study was done in BP Koirala institute of health sciences, Dharan, Nepal. It was a single center cross sectional observational study. The objective of the study was to study the prevalence and pattern of iron deficiency in heart failure with reduced ejection fraction patients. Patients coming to the cardiology opd or emergency and getting admitted to the cardiology ward or CCU and willing to give consent for the study were included in the study. All consecutive patients having clinical criteria for heart failure (according to the Framingham criteria), who were 18 years or older in age and were in NYHA functional class II- IV at the time of enrolment were included in this study. They were included regardless of the presence of anemia. Those who had co morbid non cardiac conditions causing ID (e.g. hemorrhoids, malignancy, etc.) or confounding assignment of etiology for fluid overload (e.g. end-stage renal failure), as well as patients with specific etiologies (e.g. congenital heart disease), who would be expected to follow a different natural history compared with a 'typical' HF patient were excluded from the study. All participants underwent thorough history (including dietary history) and clinical evaluation, blood sampling, and comprehensive transthoracic echocardiography using Phillips iE33 ultrasound machine and X5 sonography probe.

\section{Operational definition of anemia and iron deficiency and heart failure with reduced ejection fraction:}

Serum ferritin $<100 \mu \mathrm{g} / \mathrm{l}$ was used to diagnose absolute ID. Functional ID was defined as a serum ferritin level of 100-300 $\mu \mathrm{g} / \mathrm{l}$ and a transferrin saturation of $<20 \%$.[1] Anemia was defined as hemoglobin ( $\mathrm{Hb}$ ) $<13 \mathrm{~g} / \mathrm{dl}$ for males and $<12 \mathrm{~g} / \mathrm{dl}$ for females, based on World Health Organization definition. Patients were characterized as having normal Ejection Fraction $(\mathrm{EF} \geq 50 \%)$ or mild (EF 45-50\%), moderate (EF 31$44 \%)$, or severe (EF $\leq 30 \%)$ LV systolic dysfunction.

Outcome variables: Hemoglobin concentration, serum iron level, total iron binding capacity, transferring saturation \%, serum ferritin level, ejection fraction

Statistical analysis: The sampling method was random non probability purposive sampling. Using SPSS software tabulations and cross tabulations were done and values expressed as mean plus minus standard deviation. Using the prevalence (P) of iron deficiency in heart failure to be 30 to $40 \%$ as shown by previous studies,[8] and using $\mathrm{Z}$ value of 1.96 for a confidence interval of $95 \%$ 
and precision of $10 \%, d$ value of 0.05 the estimated sample size was 60 patients. Categorical variables are expressed in numbers and percentages, whereas normally distributed data are expressed as mean \pm standard deviation. Chi-square test and Student's $t$-test was used to calculate $p$ value as appropriate.

\section{RESULTS}

Total of 60 patients were studied out of which 41 were males and 19 were females. Mean age of the study subjects was $64.52 \pm 14.69$ years, with mean NYHA class of $2.82 \pm 0.87$ and mean EF of $25.91 \pm 10.39 \%$. Baseline characteristics of these patients are shown in Table 1.

\begin{tabular}{|c|c|}
\hline $\begin{array}{l}\text { Total patients } \\
\text { Male } \\
\text { Female }\end{array}$ & $\begin{array}{l}60 \\
41(68.33 \%) \\
19(31.66 \%)\end{array}$ \\
\hline Mean age & $64.52 \pm 14.69$ years \\
\hline Ischemic Cause & $32(53.33 \%)$ \\
\hline $\begin{array}{l}\text { NYHA Class } \\
\text { Class II } \\
\text { Class III } \\
\text { Class IV }\end{array}$ & $\begin{array}{l}23(38.33 \%) \\
22(36.66 \%) \\
15(25.00 \%)\end{array}$ \\
\hline $\begin{array}{l}\text { Ejection Fraction } \\
\text { LVEF < 30\% } \\
\text { LVEF } 31-44 \% \\
\text { LVEF } 45-50 \%\end{array}$ & $\begin{array}{l}42(70.00 \%) \\
14(23.33 \%) \\
4(6.7 \%)\end{array}$ \\
\hline Anemia & $29(48.33 \%)$ \\
\hline No Anemia & $31(51.67 \%)$ \\
\hline
\end{tabular}

\begin{tabular}{|l|l|}
\hline $\begin{array}{l}\text { Table 2: } \\
\text { Mean Value (n=60) }\end{array}$ \\
\hline Serum iron concentration & $49.72 \pm 28.93 \mu \mathrm{g} / \mathrm{L}$ \\
\hline $\begin{array}{l}\text { Serum ferritin } \\
\text { concentration }\end{array}$ & $342.08 \pm 444.63 \mu \mathrm{g} / \mathrm{L}$ \\
\hline TIBC & $208.43 \pm 73.83 \mu \mathrm{g} / \mathrm{L}$ \\
\hline Transferrin saturation & $26.24 \pm 21.01 \%$ \\
\hline Hemoglobin & $12.26 \pm 3.20 \mathrm{gm} / \mathrm{dl}$ \\
\hline
\end{tabular}

The mean serum iron concentration was
$49.72 \pm 28.93 \mu \mathrm{g} / \mathrm{L}$, Serum ferritin concentration was $342.08 \pm 444.63 \mu \mathrm{g} / \mathrm{L}$, Mean TIBC was $208.43 \pm 73.83 \mu \mathrm{g} / \mathrm{L}$ and the mean transferrin saturation was $26.24 \pm$ $21.01 \%$. Iron deficiency was found in 28 (46.67\%) patients. Absolute iron deficiency was present in 22 patients (36.67\%). Functional iron deficiency was present in 6 (10\%) patients. $29(48.33 \%)$ patients were anemic.

\begin{tabular}{|l|c|c|}
\hline $\begin{array}{l}\text { Table 3. Status } \\
\text { population (n=6) }\end{array}$ & $\begin{array}{c}\text { Absolute iron } \\
\text { deficiency } \\
(\mathbf{n = 2 2}) \\
(\mathbf{3 6 . 6 7 \% )}\end{array}$ & $\begin{array}{c}\text { Functional iron } \\
\text { deficiency } \\
(\mathbf{n = 6 )} \\
\mathbf{( 1 0 \% )}\end{array}$ \\
\hline Males & $12(54.54 \%)$ & $4(66.67 \%)$ \\
\hline Females & $10(45.45 \%)$ & $2(33.33 \%)$ \\
\hline Anemia & $11(50.00 \%)$ & $2(33.33 \%)$ \\
\hline No anemia & $11(50.00 \%)$ & $4(66.67 \%)$ \\
\hline NYHA Class & $8(\mathrm{p}>0.05)$ & $3(\mathrm{p}>0.05)$ \\
I/II & $8(\mathrm{p}>0.05)$ & $2(\mathrm{p}>0.05)$ \\
III & $6(\mathrm{p}>0.05)$ & $1(\mathrm{p}>0.05)$ \\
IV & $19(\mathrm{p}>0.05)$ & $5(\mathrm{p}>0.05)$ \\
\hline Ejection & $2(\mathrm{p}>0.05)$ & $1(\mathrm{p}>0.05)$ \\
\hline Fraction & $1(\mathrm{p}>0.05)$ & $0(\mathrm{p}>0.05)$ \\
LVEF < than & & \\
30\% & & \\
LVEF 31 - 44\% & & \\
LVEF 45-50\% & & \\
\hline
\end{tabular}

${ }^{*} \mathrm{p}$ Value less than 0.05 for statistical significance

Among the anemic subjects, ID was present in 13 (44.83\%). Absolute iron deficiency was present in 11 (37.9\%) cases in the anemic group while functional iron deficiency was present in $2(6.9 \%)$ in the anemic group. In the non-anemic group, ID was present in 15 (48.38\%). Absolute ID was present in 11 (35.5\%) patients and functional iron deficiency was present in $4(12.9 \%)$ patients in the non anemic group. Females had a higher prevalence of ID compared to males (63.16\% vs $39.02 \%)$ although the difference did not reached statistical significance $(p>0.05)$. Patients with ID with anemia were stratified as per their $\mathrm{Hb}$ level. Majority $(89.7 \%)$ of these patients were having mild anemia (Hb: $10-13 \mathrm{~g} / \mathrm{dl}$ for males and $10-$ 
$12 \mathrm{~g} / \mathrm{dl}$ for females). Patients with ID were further categorized as per their NYHA functional class and Left ventricular systolic function (as assessed by ejection fraction). The mean NYHA class in the iron deficiency group was 2.78 and the ejection fraction was 23.39\%. Absolute ID had mean Ejection fraction of $24.09 \%$ and NYHA class of 2.81 . Those with functional ID had a mean ejection fraction of 2.67, and mean ejection fraction of $20.83 \%$. Among patients without iron deficiency the mean ejection fraction was $28.12 \%$ and the mean NYHA class was 2.84 .

\section{DISCUSSION}

It was recently shown that patients with chronic HF are more susceptible to become iron deficient. This could be explained by gradual depletion of iron stores (absolute ID) due to low iron intake, gastrointestinal blood loss, or iron malabsorption [9]. Chronic inflammation, commonly observed in chronic $\mathrm{HF}$, may also play a role. Inflammation causes reduced iron absorption and availability of iron recycled in the reticulo-endothelial system (functional ID)[10]. Therefore functional iron deficiency can occur despite adequate iron stores.

The findings from this study highlight a high prevalence of ID in HF patients (46.67\%). ID is prevalent in $\mathrm{HF}$ patients even without anemia (48.38\%), which is already an established poor prognostic factor. Opasich et al [11] reported that among 148 patients with chronic HF and anemia, impaired iron supply was the cause in nearly all patients with anemia of chronic disease. In a large observational trial by Jankowska et al, [12] ID was present in $37 \%$ of all systolic chronic HF patients. In another recent study, Parikh et al [13] reported a prevalence of $61 \%$ among community-dwelling HF patients.
In this study the prevalence of iron deficiency was similar between the anemic and non anemic subjects (44.83\% vs $48.38 \%$, p>0.05). While absolute iron deficiency was more prevalent in anemic subjects as compared to non anemic subjects $(37.9 \%$ vs $35.5 \%)$, functional iron deficiency was more prevalent in non anemic subjects ( $12.9 \%$ vs $6.9 \%)$ although the differences did not reach statistical significance $(p>0.05)$.

Female gender had a higher prevalence of iron deficiency (63.16\% vs $39.02 \%)$. This finding is in accordance with the results of previous studies which has shown female gender to be an independent correlate of iron deficiency in heart failure [14]. The mean age of the study population in this study was 64.52 years, so it is unlikely that the increased iron deficiency in the female subjects was due to blood loss due to menstruation since all of the female subjects were post menstrual.

The data to support the predictive value of iron deficiency for predicting mortality in heart failure is conflicting and this study was not intended to study the predictive value or the influence of iron deficiency on survival or prognosis of heart failure. Over the years, anemia has been associated with an adverse outcome in patients with chronic HF [15]. The mechanism by which anemia contributes to an adverse outcome in these patients is complex and multifactorial [16] Jankowska et al [12] examined 546 patients with mild to severe systolic chronic HF. In both univariate and multivariate analyses, ID-but not anemia-was an independent predictor of allcause mortality or heart transplantation. [12] Okonko et al [17] identified ID as a predictor of elevated mortality in 157 chronic $\mathrm{HF}$ patients, independent of hemoglobin level. In contrast, Parikh et al [13] found that ID was 
not associated with all-cause or cardiovascular mortality in 574 patients with self-reported HF. Till date it is unknown whether it is anemia that contributes to adverse prognosis or whether one of the factors contributing factors to anemia-such as inflammation-also contributes to adverse outcome [18].

\section{CONCLUSION}

Iron deficiency is an emerging problem in chronic HF, affecting almost half of the patients. This study highlights the underestimated and neglected burden of iron deficiency in heart failure patients. Further large scale randomized studies should be carried out to study the patho-physiological mechanisms of iron deficiency in heart failure patients and the effect on hard cardiovascular outcomes in heart failure patients due to iron deficiency and the effects of iron supplementation. Prognostic models should be developed incorporating markers of iron deficiency in heart failure patients.

\section{REFERENCES}

1. Jankowska EA, von Haehling S, Anker SD, Macdougall IC, Ponikowski P. Iron deficiency and heart failure: diagnostic dilemmas and therapeutic perspectives. European heart journal. 2013 Mar;34(11):816-29. PubMed PMID: 23100285. Pubmed Central PMCID: Pmc3596759. Epub 2012/10/27. eng.

2. Jankowska EA, Rozentryt P, Witkowska A, et al. Iron deficiency predicts impaired exercise capacity in patients with systolic chronic heart failure. Journal of cardiac failure. 2011 Nov;17(11):899-906. PubMed PMID: 22041326. Epub 2011/11/02. eng.

3. von Haehling S, Ebner N, Evertz R, Ponikowski $\mathrm{P}$, Anker SD. Iron Deficiency in Heart Failure. An Overview. 2019;7(1):36-46.

4. Rangel I, Gonçalves A, De Sousa C, et al. Iron deficiency status irrespective of anemia: a predictor of unfavorable outcome in chronic heart failure patients. Cardiology. 2014;128(4):320-6.

5. Ponikowski P, Voors AA, Anker SD, et al. 2016 ESC Guidelines for the diagnosis and treatment of acute and chronic heart failure: The Task Force for the diagnosis and treatment of acute and chronic heart failure of the European Society of Cardiology (ESC)Developed with the special contribution of the Heart Failure Association (HFA) of the ESC. European Heart Journal. 2016;37(27):2129-200.

6. Charles-Edwards G, Amaral N, Sleigh A, et al. Effect of Iron Isomaltoside on Skeletal Muscle Energetics in Patients With Chronic Heart Failure and Iron Deficiency. Circulation. 2019 May 21;139(21):2386-98. PubMed PMID: 30776909. Epub 2019/02/20. eng.

7. Moliner P, Enjuanes C, Tajes M, et al. Association Between Norepinephrine Levels and Abnormal Iron Status in Patients With Chronic Heart Failure: Is Iron Deficiency More Than a Comorbidity? Journal of the American Heart Association. 2019 Feb 19;8(4):e010887. PubMed PMID: 30760082. Pubmed Central PMCID: Pmc6405646. Epub 2019/02/15. eng.

8. Cohen-Solal A, Leclercq C, Deray G, et al. Iron deficiency: an emerging therapeutic target in heart failure. Heart. 2014;100(18):1414-20.

9. Alexandrakis MG, Tsirakis G. Anemia in heart failure patients. ISRN Hematol. 2012;2012:246915-. PubMed PMID: 22536520. Epub 03/25. eng.

10. Weiss G, Goodnough LT. Anemia of chronic disease. The New England journal of medicine. 2005 Mar 10;352(10):1011-23. PubMed PMID: 15758012. Epub 2005/03/11. eng.

11. Opasich C, Cazzola M, Scelsi L, et al. Blunted erythropoietin production and defective iron supply for erythropoiesis as major causes of anaemia in patients with chronic heart failure. Eur Heart J. 2005 Nov;26(21):2232-7. PubMed PMID: 15987710. Epub 2005/07/01. eng.

12. Jankowska EA, Rozentryt P, Witkowska A, et al. Iron deficiency: an ominous sign in patients with systolic chronic heart failure. Eur Heart J. 2010 Aug;31(15):1872-80. PubMed PMID: 20570952. Epub 2010/06/24. eng. 
13. Parikh A, Natarajan S, Lipsitz SR, Katz SD. Iron deficiency in community-dwelling US adults with selfreported heart failure in the National Health and Nutrition Examination Survey III: prevalence and associations with anemia and inflammation. Circ Heart Fail. 2011;4(5):599-606. PubMed PMID: 21705484. Epub 06/24. eng.

14. Klip IT, Comin-Colet J, Voors AA, et al. Iron deficiency in chronic heart failure: an international pooled analysis. American heart journal. 2013 Apr;165(4):575-82 e3. PubMed PMID: 23537975. Epub 2013/03/30. eng.

15. Groenveld HF, Januzzi JL, Damman K, et al. Anemia and mortality in heart failure patients: a systematic review and meta-analysis. Journal of the American College of Cardiology. 2008;52(10):818-27.

16. van der Meer P, van Veldhuisen DJ. Anaemia and renal dysfunction in chronic heart failure. Heart. 2009;95(21):1808-12.

17. Okonko DO, Mandal AK, Missouris CG, PooleWilson PA. Disordered iron homeostasis in chronic heart failure: prevalence, predictors, and relation to anemia, exercise capacity, and survival. Journal of the American College of Cardiology. 2011;58(12):1241-51.

18. Kalantar-Zadeh K, Kalantar-Zadeh K, Lee GH. The fascinating but deceptive ferritin: to measure it or not to measure it in chronic kidney disease? Clinical journal of the American Society of Nephrology : CJASN. 2006 Sep;1 Suppl 1:S9-18. PubMed PMID: 17699375. Epub 2007/09/19. eng

\section{Correspondence to:}

Dr. A. Mahaseth

Department of Cardiology, BP Koirala Institute of Health Sciences, Dharan, Nepal

amahaseth@hotmail.com 